\title{
Helicoidal peripapillary chorioretinal degeneration: electrophysiology and psychophysics in 17 patients
}

\author{
Thor Eysteinsson, Fridbert Jónasson, Vésteinn Jónsson, Alan C Bird
}

\begin{abstract}
Aims-To characterise retinal function using electrophysiological and psychophysical tests in 17 patients with helicoidal peripapillary chorioretinal degeneration. Methods-The electroretinogram (ERG) was recorded using gold foil corneal electrodes. The electro-oculogram (EOG) was recorded using a standard protocol. Dark adaptometry was recorded with an SST-1 dark adaptometer and colour vision assessed with Ishihara plates and Farnsworth D-15.

Results-All subjects had a recordable ERG. The amplitudes and implicit times of the $a-$ and $b$-waves were within normal limits at all luminances in five subjects (age 21-70 years, mean 40 years). The ERG of six (age 26-55 years, mean 40.7 years) had subnormal amplitudes at all luminances, but normal implicit times, and six (age 38-81 years, mean 60.7 years) had abnormal ERGs with marked reduction of a- and b-waves, and delayed implicit times of the b-wave. The implicit times of the a-wave were normal in all subjects. A reduction in the b/a wave ratios was not found, nor was there selective loss of scotopic, mixed rod/cone, or cone responses. The light/dark ratio of the EOG was subnormal (150-185\%) or abnormal (below $150 \%$ ) in all but three subjects. Two patients with normal EOG showed normal ERGs in both eyes, but one had subnormal ERGs in both eyes. The scotopic sensitivity was normal in all subjects and dark adaptation showed a normal time course. Colour vision was normal in all patients.

Conclusion-The results suggest that in most cases the function of the retinal pigment epithelium is affected by this disease before any changes in the function of the sensory retina are detectable by our methods, and that retinal dysfunction is focal rather than diffuse.

(Br f Ophthalmol 1998;82:280-285)
\end{abstract}

Moorfields Eye Hospital, University of London, City Road, London

Alan C Bird

Correspondence to: Thor Eysteinsson, Department of Physiology, University of Iceland, Vatnsmyrarvegi 16, 101 Reykjavik, Iceland.

Accepted for publication 28 October 1997
Sveinsson and by Francescetti, clinical photographs were not presented, which has led to some confusion regarding the terminology used in the literature. However, this has been illustrated in more recent reports on the same patients. $^{25}$ We have kept a register of Icelandic patients for the past 15 years, and this now includes 102 individuals, all of whom have common ancestors in north eastern Iceland in the early 18 th century. Clinical findings include lesions of the fundi that are always bilateral, with tongue-like, well defined strips of chorioretinal atrophy radiating from the optic nerve head. These lesions have been seen as early as 6 months of age, are of variable size in childhood, and enlarge slowly during life. Separate, mostly circular lesions may also be found in the periphery. As demonstrated by fundus photography and fluorescein angiography it appears that the pigment epithelium and the choriocapillaris are affected initially, with later involvement of the larger choroidal vessels and the sensory retina. ${ }^{5-7} \mathrm{~A}$ recent study on 20 of our patients suggests that the mutations responsible for these changes are localised on chromosome $11 \mathrm{p} 15 .{ }^{8}$

To date, limited attempts have been made to examine the functional changes that accompany HPCD, and the only reports of electrophysiological examinations present in the literature are electroretinogram (ERG) recordings from one patient. ${ }^{5}$ The present report summarises our initial electrophysiological findings from 17 patients with HPCD, using the clinical flash ERG and the electrooculogram (EOG), in addition to results obtained by dark adaptometry and assessment of colour vision.

Materials and methods

The subjects in this study were recruited among patients known to be affected by the disease in Iceland, the majority living in Reykjavik or its vicinity. They were contacted through their respective ophthalmologists, who explained to them the basic objectives of the study. On the basis of fundus photographs, patients with lesions of variable size were approached, care being taken to select a representative sample of age, sex, and severity of disease. All those approached agreed to participate, with one exception. The age range was 21-81 years, and there were nine men and eight women. The disease range from very mild to widespread degeneration of the choroid, the pigment epithelium, and the neurosensory retina. Figures $1 \mathrm{~A}, \mathrm{~B}$, and $\mathrm{C}$ show fundus photographs, taken from a father, his son, and a grandson. All of our patients have subsequently been linked to chromosome $11 \mathrm{p} 15 .^{8}$ 

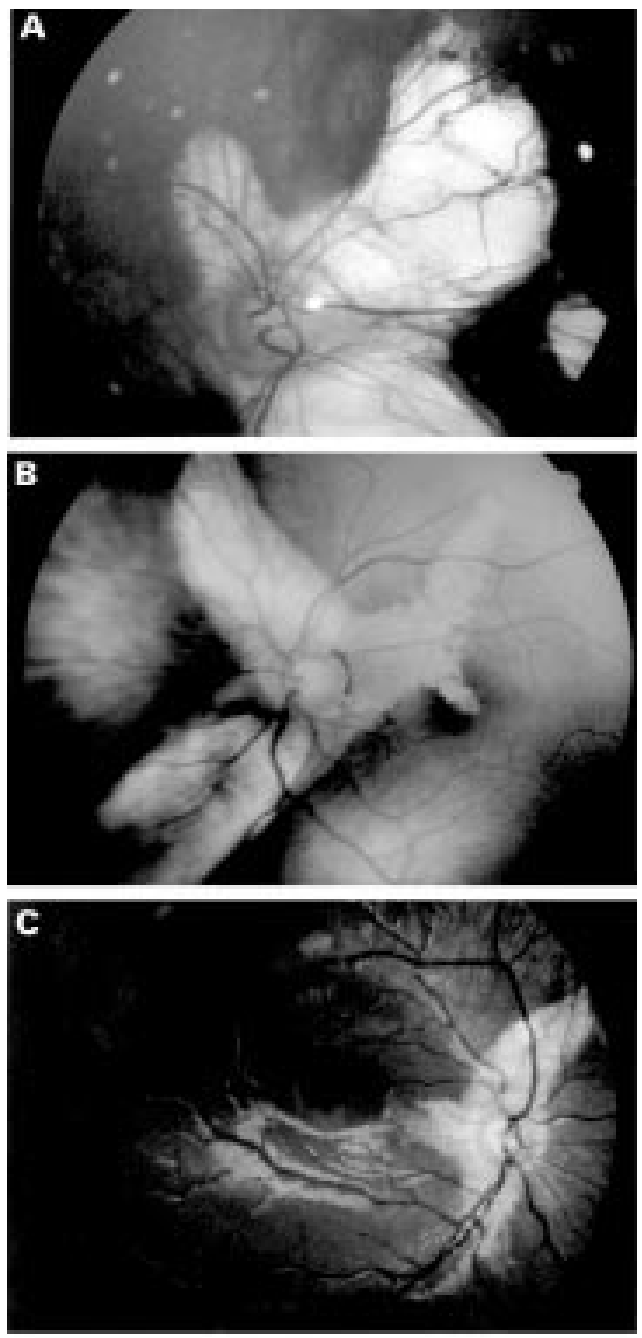

Figure 1 (A) The father, 72 years of age, with extensive peripapillary lesions extending horizontally and also vertically into the macula. (B) His son, 45 years of age, with starfish-like lesions extending from the disc, macula intact. $(C)$ The 3 year old grandson (spared electrophysiology) with peripapillary lesions, one tongue extending towards the macula.

Values were established for normal subjects $(n=8)$ who volunteered for electrophysiological and psychophysical testing. The age range of the normal volunteers was comparable with that of the patients (19-75 years). All patients and normal subjects gave informed consent to participate. All procedures used in the present study conform to the Declaration of Helsinki. The ERG values from both eyes of subjects for each stimulus condition were averaged and the significance of the results was examined with a Student's $t$ test of means between independent groups.

The flash ERGs were recorded with a standard preamplifier and averaging system. Before ERG recording, both pupils were dilated with $1 \%$ tropicamide. The ERG was recorded with gold foil corneal electrodes ${ }^{9}$ placed in the lower fornix and EEG gold cup skin electrodes as reference placed on the temple. A gold cup skin electrode placed on the forehead acted as an earth. The preamplifier bandwidth was set at $0.1-300 \mathrm{~Hz}$. After dark adaptation of at least 30 minutes, a protocol of test stimuli was pre- sented to the patients in a Ganzfeld system. The protocol was similar to a recently proposed international standard, ${ }^{10}$ except that the oscillatory potentials were not recorded, and subjects were not light adapted with background light, so as to shorten the time necessary to perform ERG testing on each subject. Scotopic ERGs were obtained after at least 30 minutes of dark adaptation using stimuli presented at a repetition rate of $0.2 \mathrm{~Hz}$. Each scotopic ERG presented in this paper is an average of a maximum of five stimulus presentations. After dark adaptation Ganzfeld stimuli were first presented with a Kodak Wratten gelatine filter no 47 (blue, maximum transmission measured at $437 \mathrm{~nm}$ ) placed in the light path, and the luminance attenuated with neutral density filters to $-2.9 \log \mathrm{cd} . \mathrm{s} / \mathrm{m}^{2}$. The luminance of these stimuli was then increased in fixed steps by removal of neutral density filters. Mixed cone-rod ERGs were obtained by white stimuli $\left(-0.27 \log \mathrm{cd} . \mathrm{s} / \mathrm{m}^{2}\right)$ at $0.2 \mathrm{~Hz}$ repetition rate, and the proportionate contribution of rods to the response was reduced by increasing the repetition rate of white stimuli of maximum luminance $(1.2 \mathrm{log}$ cd.s $/ \mathrm{m}^{2}$ ) to $2 \mathrm{~Hz}$. Each mixed cone-rod ERG recording presented in this paper is an average of a maximum of 20 repetitions. Finally, cone ERG responses to $31 \mathrm{~Hz}$ white flicker were recorded, with rejection of responses to the first 2 seconds of stimulus presentation before averaging.

The EOG was recorded according to a published protocol. ${ }^{11}$ Briefly, skin electrodes were placed at the lateral canthi and potential between these recorded using an AC amplifier. The patient was placed in a Ganzfeld and asked to undertake lateral saccades of 30 degrees at regular intervals for 15 minutes in the dark, and then similarly for 15 minutes with the Ganzfeld illuminated with $1.24 \mathrm{log}$ $\mathrm{cd} / \mathrm{m}^{2}$ white light. Pupils were not dilated and there was no preadaptation period before testing. A light/dark (L/D) ratio of $185 \%$ or higher is normal in this laboratory, below $150 \%$ is designated abnormal and intermediate values are designated as subnormal.

Dark adaptometry was performed with a portable SST-1 dark adaptometer (LKC Technologies, Gaithersburg, USA) equipped with a hand held dome for presentation of a light adaptation $\left(1050 \mathrm{~cd} / \mathrm{m}^{2}\right)$ for 60 seconds, and for presentation of test stimuli (full field with a dominant wavelength of $575 \mathrm{~nm}$ ). A forced choice procedure was used for threshold (expressed in decibels $(\mathrm{dB})$, where $30 \mathrm{~dB}=$ $-2.35 \log \mathrm{cd} / \mathrm{m}^{2}$ ) measurements. They were taken at intervals of about 30 seconds during 30 minutes of dark adaptation, beginning immediately after cessation of the bleach. The method for dark adaptometry used primarily measures the scotopic phase of dark adaptation, due to the selective spectral composition of the test stimuli, and the maximum luminance, which is about $2.5 \log _{10}$ lower than from Goldmann-Weekers type of dark adaptometers. Colour vision was assessed by administering the Ishihara pseudoisochromatic plates and the Farnsworth D-15 hue test to the 
Table 1 A summary of ERG results in 17 patients with helicoidal peripapillary chorioretinal degeneration

\begin{tabular}{|c|c|c|c|c|c|c|c|c|c|c|}
\hline \multirow[b]{3}{*}{ Patient no } & \multirow[b]{3}{*}{ Age } & & & \multirow{3}{*}{$\begin{array}{l}\text { Scotopic } \\
b \text {-wave } \\
(\mu V)^{\star}\end{array}$} & \multirow{3}{*}{$\begin{array}{l}\text { Mixed rod/cone } \\
b \text {-wave }(\mu V)^{\star}\end{array}$} & \multirow{3}{*}{$\begin{array}{l}\text { Flicker } \\
(\mu V)^{\star}\end{array}$} & \multirow{3}{*}{$\begin{array}{l}\text { Rod/cone } \\
\text { b/a ratios }\end{array}$} & \multicolumn{3}{|c|}{ Implicit times (ms) } \\
\hline & & \multicolumn{2}{|l|}{ Acuity } & & & & & \multicolumn{2}{|c|}{ Mixed rod/cone } & \multirow{2}{*}{$\frac{\text { Scotopic }}{\text { a-wave }}$} \\
\hline & & Left eye & Right eye & & & & & $b$-wave & $a$-wave & \\
\hline 1 & 29 & $20 / 20$ & $20 / 15$ & 230 & 316 & 90 & 2.5 & 33 & 22 & 28 \\
\hline 2 & 38 & $20 / 60$ & $20 / 400$ & 150 & 170 & 50 & 2.21 & 44 & 26 & 32 \\
\hline 3 & 21 & $20 / 20$ & $20 / 20$ & 391 & 406 & 90 & 2.3 & 34 & 23 & 28 \\
\hline 4 & 71 & $\mathrm{CF}$ & $20 / 70$ & 187 & 231 & 42 & 2.3 & 46 & 30 & 35 \\
\hline 5 & 79 & $20 / 120$ & $\mathrm{CF}$ & 161 & 169 & 24 & 2.35 & 48 & 32 & 35 \\
\hline 6 & 81 & $20 / 80$ & $20 / 200$ & 191 & 234 & 5 & 2.12 & 40 & 29 & 35 \\
\hline 7 & 55 & $20 / 20$ & $20 / 20$ & 291 & 281 & 86 & 3.57 & 42 & 24 & 31 \\
\hline 8 & 26 & $20 / 20$ & $20 / 20$ & 247 & 228 & 78 & 2.38 & 37 & 27 & 30 \\
\hline 9 & 24 & $20 / 20$ & $20 / 200$ & 303 & 394 & 78 & 2.02 & 36 & 25 & 30 \\
\hline 10 & 70 & $20 / 40$ & $20 / 40$ & 275 & 331 & 94 & 2.25 & 40 & 28 & 34 \\
\hline 11 & 46 & $20 / 20$ & $20 / 20$ & 309 & 268 & 75 & 1.93 & 36 & 24 & 29 \\
\hline 12 & 53 & $20 / 30$ & $20 / 30$ & 272 & 284 & 68 & 2.1 & 36 & 27 & 31 \\
\hline 13 & 70 & $20 / 120$ & $20 / 120$ & 122 & 109 & 69 & 2.8 & 38 & 29 & 31 \\
\hline 14 & 42 & $20 / 80$ & $20 / 80$ & 150 & 172 & 63 & 2.07 & 46 & 29 & 34 \\
\hline 15 & 29 & $20 / 200$ & $20 / 200$ & 231 & 250 & 11 & 1.95 & 32 & 22 & 31 \\
\hline 16 & 50 & $20 / 20$ & $20 / 20$ & 319 & 375 & 72 & 2.45 & 39 & 25 & 31 \\
\hline 17 & 51 & $20 / 20$ & $20 / 20$ & 144 & 244 & 31 & 2.1 & 40 & 24 & 30 \\
\hline Patient mean & & & & 233 & 262 & 60 & 2.33 & 39.2 & 24 & 29 \\
\hline SD & & & & 76 & 83 & 28 & 0.38 & 4.72 & 2.71 & 1.41 \\
\hline Normal range $(\mu \mathrm{V})$ & & & & $200-456$ & $340-680$ & $82-125$ & $2.05-2.75$ & & & \\
\hline Normal mean $(\mu \mathrm{V})$ & & & & 342 & 437 & 98 & 2.33 & 39 & 22.9 & 2.8 \\
\hline SD & & & & 69 & 97 & 13 & 0.22 & 4.6 & 0.87 & 1.3 \\
\hline
\end{tabular}

* The flicker response was evoked with a repetition rate of $31 \mathrm{~Hz}$ at maximum intensity $\left(-0.19 \mathrm{log} \mathrm{cd} . \mathrm{s} / \mathrm{m}^{2}\right)$, the scotopic b-wave at the maximum intensity used for eliciting rod responses $\left(-1.22 \log \mathrm{cd} . \mathrm{s} / \mathrm{m}^{2}\right)$, and the mixed rod-cone b-wave at $-0.27 \log \mathrm{cd} . \mathrm{s} / \mathrm{m}^{2}$ intensity.

patients illuminated by a C-source. Both tests were administered under monocular viewing conditions.

\section{Results}

All the patients examined in this study have classic HPCD phenotype. Both eyes were always affected. The lesions radiating from the optic disc were of variable size and extent. The present study was undertaken to examine the

Scotopic blue ( $\min$ ) $\left(-2.6 \log \mathrm{cd} . \mathrm{s} / \mathrm{m}^{2}\right)$
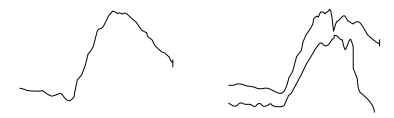

Scotopic blue (max) $\left(-1.22 \log \mathrm{cd} . \mathrm{s} / \mathrm{m}^{2}\right)$
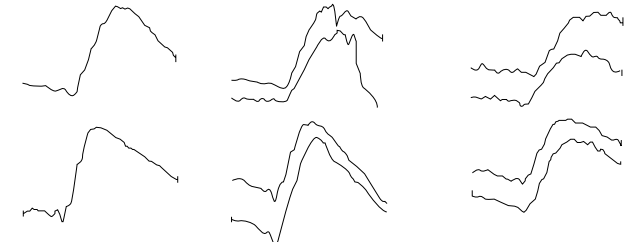

Mixed cone-rod response $\left(-0.27\right.$ log cd.s $\left./ \mathrm{m}^{2}\right)$
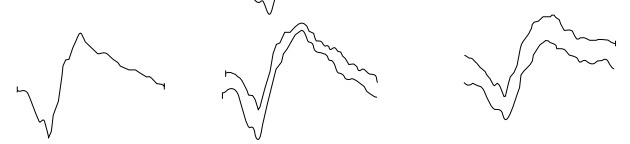

Mixed cone-rod response (1.2 log cd.s $/ \mathrm{m}^{2}$ )
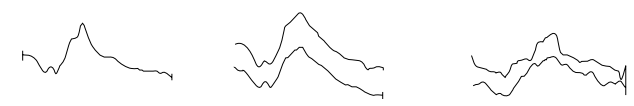

$31 \mathrm{~Hz}$ white flicker $\left(-0.19\right.$ log cd.s $\left./ \mathrm{m}^{2}\right)$

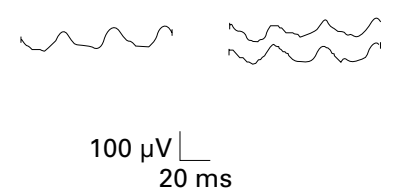

$20 \mathrm{~ms}$

Figure 2 A short version of the ERG protocol, taken from a normal individual, with an EOG L/D ratio of $280 \%$ on each eye (left column of records), and from two patients with HPCD showing normal ERGs. The ERGs in the middle column of the figure are from a 26 year old female with 20/20 vision and normal EOGs. The records in the column on the right are from a 70 year old male with $20 / 40$ vision in both eyes and abnormal EOGs. The top row of ERG records was obtained in response to the lowest luminance used, after 30 minutes of dark adaptation, and with a Wratten 47 (blue) gelatin filter placed in the light path. The second row is in response to blue stimuli of the highest luminance used, where a clear rod $a$ - and $b$-waves are found. The middle row shows responses to scotopic white stimuli of the highest luminance used, and a mixed rod and cone contribution to the response. The second lowest row of recordings shows mixed rod-cone responses to white light of maximum intensity and presented at a repetition rate of $2 \mathrm{~Hz}$. The lowest row of recordings were obtained in response to $31 \mathrm{~Hz}$ white flicker. Stimulus onset occurred at the start of each trace. The calibration marker at bottom applies to all records in this figure.

functional consequences of HPCD lesions, and to attempt to relate the changes in function to the age of patients and the extent of the lesions.

The amplitudes and implicit times of the ERG varied widely (Table 1). Five of the patients (age 23-70 years, mean 41 years) had normal ERG amplitudes and implicit times of the a- and b-waves at all luminances (Fig 2).

The amplitude of the ERGs of six patients (age 26-71 years, mean 44 years) was subnormal at some or all luminances used, while implicit times were within the normal range. The third group (six patients, age $38-81$ years, mean 60.7 years) showed a marked reduction of a- and b-wave amplitudes and significantly prolonged b-wave implicit times (Fig 3).

Although the values demonstrate variability of the a-waves and b-waves, and flicker amplitudes among the HPCD patients, the overall means are significantly lower than among normal subjects. The mean amplitude of the scotopic a-waves (Table 1) of the patients as a group (elicited at maximum light intensity) was significantly reduced compared with normal amplitude (independent $t$ test, $\mathrm{t}(25)=3.03$, $\mathrm{p}<0.005$ ), as well as that of the mixed cone-rod a-waves $(\mathrm{t}(25)=2.79, \mathrm{p}<0.01)$.

However, the mean implicit times of the scotopic a-waves did not differ significantly between patients and normal subjects $(\mathrm{t}(25)=1.68, \mathrm{p}=0.10)$, nor did the mixed conerod a-wave implicit times $(\mathrm{t}(25)=1.66$, $\mathrm{p}=0.10)$. As the $t$ test assumes equal standard deviations of populations, and the standard deviations of the mean implicit times of mixed cone-rod a-wave responses were found to differ significantly $(F=9.64, p<0.001)$, the data were submitted to a non-parametric test, but the difference between means was still found to be not significant (Mann-Whitney test, $\mathrm{p}<0.1$ ). Similarly, the mean amplitude of the scotopic b-waves of the patients as a group was significantly reduced $(\mathrm{t}(25)=3.68, \mathrm{p}<0.001)$, as well as the amplitudes of the mixed cone-rod 


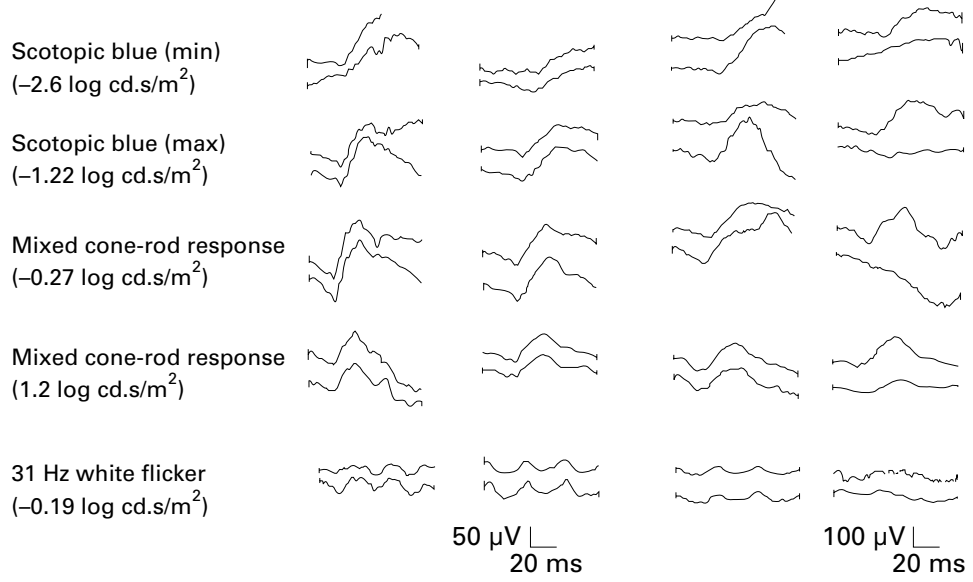

Figure 3 The subnormal ERGs of two patients with HPCD (patients 15 (left column) and 4 (second column from left) in Table 1), and the abnormal ERGs of two patients with HPCD (patients 1 (third column from left) and 13 (right column) in Table 1), in response to the same stimuli as in Figure 2. The upper recordings in each pair of figures were obtained from the left eye. The calibration marker on the bottom right applies to all recordings in the figure, except that the amplitude marker labelled " $50 \mu V$ " applies to the $31 \mathrm{~Hz}$ white flicker responses above it.

b-waves $(\mathrm{t}(25)=4.94, \mathrm{p}<0.0001)$ and $31 \mathrm{~Hz}$ flicker $(\mathrm{t}(25)=3.93, \mathrm{p}<0.0006)$, all evoked by maximum intensity of light stimuli. The standard deviations of flicker amplitudes differed significantly $(\mathrm{F}=4.72, \mathrm{p}<0.01)$, but the mean amplitudes were still found to be significantly different on a non-parametric test (MannWhitney, $\mathrm{p}<0.0003$ )

The mean implicit times of the mixed conerod b-waves (Table 1) recorded at a $0.2 \mathrm{~Hz}$ repetition rate were not significantly different between patients as a group and normal subjects $(\mathrm{t}(25)=0.15, \mathrm{p}=0.87)$, but patients with abnormal reduction of the ERG amplitude showed significantly prolonged b-wave implicit times (42.6 ms (SD 3.93)), compared with other patients (37.3 ms (4.12)) $(\mathrm{t}(15)=2.57, \mathrm{p}<0.02)$. The mean implicit time of $31 \mathrm{~Hz}$ flicker was 32 (SD 3.64) ms among the patients as a group and $30(0.8) \mathrm{ms}$ among the normal subjects, which are not significantly different $(\mathrm{t}(25)=1.699, \mathrm{p}=0.101)$. The standard deviations differed significantly $((\mathrm{F}=19.87$, $\mathrm{p}=0.0001)$, but the means were found not significantly different on a non-parametric test (Mann-Whitney, $p=0.263$ ). However, the mean flicker implicit time among the subgroup of patients with abnormal ERG amplitudes and prolonged $b$-wave implicit times was 34 (4.2) $\mathrm{ms}$, that is prolonged, although not significantly $(\mathrm{t}(25)=2.08, \mathrm{p}=0.05)$ when compared with the mean of other patients $(30.6$ (2.8) $\mathrm{ms}$ ).

The mean b/a ratio of the mixed $\mathrm{rod} /$ cone ERGs was the same among patients and normal subjects. No significant difference was found between the b/a ratios of any responses to any type of stimuli eliciting both a- and b-waves among the three groups of patients. Only one of the 17 patients deviates substantially from the overall mean, in a positive direction (patient no 7, b/a ratio: 3.57) (Table 1).

None of the ERG variables of any responses recorded correlated significantly with age. At maximum light intensity used the mixed

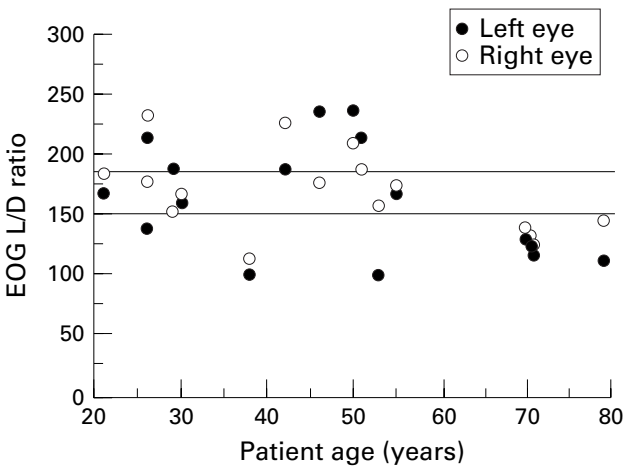

Figure 4 The light/dark ratio of the EOG as a function of age in all of the HPCD patients tested. The lower limit of the normal light rise is $185 \%$, those values below $150 \%$ are designated abnormal, while those ratios falling between these values are designated as subnormal.

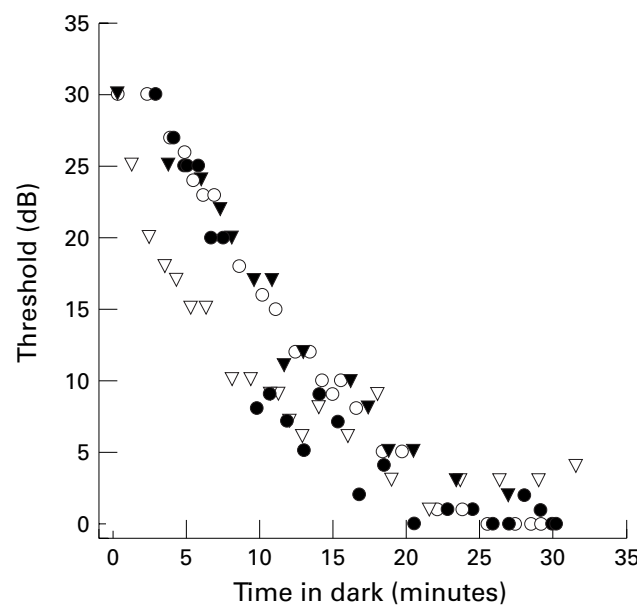

Figure 5 Dark adaptation curves for detection thresholds of whole field test stimuli, obtained from four patients with HPCD. Threshold is expressed in decibels, where $30 \mathrm{~dB}$ is equivalent to $-2.35 \log \mathrm{cd} / \mathrm{m}^{2}$. As the maximum luminance of the test stimuli were about $2.5 \log _{10}$ lower than in Goldmann-Weekers type of dark adaptometers, no rod-cone break can be observed in these curves, which are similar to dark adaptation curves obtained with the same method from normal subjects.

rod-cone b-wave correlated significantly with visual acuity (Spearman rank correlation, $r_{s}=$ $0.562, \mathrm{p}<0.01)$ as did the flicker response amplitude $\left(r_{s}=0.58, p<0.01\right)$, but the scotopic b-wave amplitude did not correlate significantly with visual acuity $\left(r_{s}=0.35, p<0.1\right)$.

All but two eyes showed a light rise of the electro-oculogram (EOG) (Fig 4). Only six eyes had normal L/D ratios ( $>185 \%$ ) and all these showed normal ERGs also, except one individual with subnormal ERGs and lesions extending into the macular region, and severely reduced flicker ERGs in both eyes. Four eyes had $\mathrm{L} / \mathrm{D}$ ratios just below or close to normal (178-184\%).

The $\mathrm{L} / \mathrm{D}$ ratio in HPCD does not correlate significantly with the age of the patients (Spearman rank correlation $r_{s}=-0.036$, $\mathrm{p}<0.1$ ), except that after the age of 60 years it is abnormal in all instances (Fig 4).

In all patients tested dark adaptation curves show a normal time course of dark adaptation and a normal final threshold even though there is a large difference in the size and extent of the lesions in their fundi.(Fig 5). 
The colour vision of all the patients, including those with lesions extending into the macular region, was found to be normal as measured by the Farnsworth D-15 hue test and the Ishihara pseudoisochromatic plates. The patients made neither more than two errors on the Ishihara plates, nor more than one placement error on the D-15 test.

\section{Discussion}

The present study is the first one on sensory function in a substantial number of patients with HPCD. Previously, ERG and EOG recordings from one patient in Switzerland were reported. ${ }^{5}$ Although no ERG recordings were shown, it appears that the ERG of this patient was comparable with those with subnormal ERG among our patients. It is clear, however, from the present work that there is a considerable variability of ERG and EOG results among patients with HPCD, ranging from normal to severely abnormal.

The normal physiological attributes in three of the patients with manifest disease implies that large portions of the RPE and the sensory retina are functionally normal and that the disease is localised in these individuals. The data from other patients indicate that the EOG is affected earlier than the ERG in most cases with this disease. Since photic stimulation is needed to elicit the light rise, the EOG results are dependent on the integrity of the sensory retina, which in some cases is clearly affected. We are currently examining RPE function in HPCD with the use of electrophysiological tests that are independent of photic stimulation and of the sensory retina. ${ }^{12}$

A reduced amplitude of the ERG was not necessarily accompanied by a prolonged implicit times of either the a- or b-waves, suggesting that in these patients the function of the photoreceptors and possibly neurons in clinically unaffected retinas is normal. In most progressive chorioretinal dystrophies such as retinitis pigmentosa there is an increase in the implicit times of a- and b-waves of the ERG as well as a decrease in amplitude, which has been attributed to abnormal phototransduction in remaining photoreceptors. ${ }^{13}$ In stationary or slowly progressively dystrophies such as sectorial retinitis pigmentosa $(\mathrm{RP})$ and patients with HPCD in the present study there is a reduction in amplitude, but not a significant increase in a-wave implicit times. Only at an advanced stage of the disease, when the amplitude is clearly abnormal, are the implicit times prolonged, and then primarily those of the b-wave. Our ERG data therefore strongly suggest that rod phototransduction is normal in unaffected areas in the retinas of HPCD patients even at an advanced stage of the disease. The presence of normal b/a ratios while the mean amplitude of a-waves and b-waves are significantly reduced suggests that loss of rod photoreceptors in affected areas of the fundi is the main cause of b-wave amplitude reduction rather than any secondary defects in b-wave generators, such as bipolar and/or glial cells. In some of the patients, with advanced disease, the implicit times of $b$-waves was prolonged while the a-wave implicit times were not. It is possible that in these cases with abnormal ERGs secondary defects in b-wave generators do occur, in addition to photoreceptor loss, which cause a delay in transmission of signals from these. The conclusion that rod photoreceptors are normal in unaffected regions is supported by psychophysical testing. However extensive the disease, all patients regained normal levels of sensitivity with normal kinetics implying that regeneration of the photopigment during the process of dark adaptation is normal.

The only cone responses recorded in the present study were evoked by white $31 \mathrm{~Hz}$ flicker. But the flicker implicit times were normal even when the amplitude was significantly reduced, except in cases with advanced disease, although not significantly. The reduction of the flicker response in some HPCD patients without an increase in the implicit time, may be due to either panretinal cone disturbances or macular lesions or both. We are currently examining macular function in this disease with other electrophysiological measures, but in view of the fact that all of our patients show normal colour vision, and normal flicker implicit times except those with the most advanced disease, the most likely explanation for the reduction in flicker amplitude is a decrease in the number of cones in some patients rather than any defect in cone phototransduction.

The changes found in the electrophysiological variables measured in this study do not appear to be correlated with age generally in HPCD, or even though the lesions have enlarged, suggesting that the functional changes may progress at a variable rate between individuals. Previous work has shown that the size of lesions seen ophthalmoscopically in this disease is not correlated with age. Severe disease may be seen in the young and mild disease in the elderly, ${ }^{1-7}$ and the present work shows that this also applies to the physiological variables. It is of interest that in spite of very substantial changes in the fundi in some cases, none of the patients has a non-recordable ERG that contrasts with RP and related diseases, and night blindness is not a complaint. Although histological evidence is lacking, it is likely that at a cellular level the changes occurring in the RPE and the sensory retina in this disease are different from those that occur in RP and similar dystrophies in that there is not widespread photoreceptor dysfunction. It should be noted finally that there are reports of similar electrophysiological attributes in pigmented paravenous retinochoroidal atrophy in the literature, ${ }^{14}$ which is clinically a more similar condition to HPCD than is RP, and has indeed been confused with HPCD.

This work was supported in part by the Icelandic Science Foundation and the Helga Jónsdóttir and Sigurlidi Kristiánsson Memorial Fund.

\footnotetext{
1 Sveinsson K. Chorioditis areata. Acta Ophthalmol 1939;17:73-80.

2 Sveinsson K. Helicoidal peripapillary chorioretinal degeneration. Acta Ophthalmol 1979;57:69-75.
} 
3 Francescetti A. A curious affection of the fundus oculi: helicoidal peripapillar chorioretinal degeneration. Its relation
to pigmentary paravenous chorioretinal degeneration. Doc to pigmentary paravenous chor

4 Magnusson L. Atropia areata, a variant of peripapillary chorioretinal degeneration. Acta Ophthalmol 1981;59:659-64.

5 Babel J. Les choroidopathies geographiques et helicoidales. Etude clinique et angiographique; essai de classification. $\mathcal{F}$ Fr Ophtalmol 1983;6:981-93.

6 Brazitikos PD, Safran AB. Helicoid peripapillary chorioretinal degeneration. Am f Ophthalmol 1990;109:290-4.

7 Jónasson F, Eysteinsson T, Jónsson V, Magnusson L, Bird AC. Helicoidal peripapillary chorioretinal degeneration. XXXII Nordic Congress of Ophthalmology, Odense, Denmark, 1995:21-24.5.

8 Fossdal R, Magnusson L, Weber JL, Jensson Ó. Mapping the locus of atrophia areata, a helicoid peripapillary chorioretinal degeneration with autosomal dominant inheritance, to chromosome 11p15. Hum Mol Gen 1995;4:47983.
9 Arden GB, Carter RM, Hogg C, Siegel IM, Margolis S. A goldfoil electrode:extending the horizons for clinical electroretinography. Invest Ophthalmol Vis Sci 1979;18: 421-6.

0 Marmor MF, Arden GB, Nilsson SE, Zrenner E. A standard for clinical electroretinography. Arch Ophthalmol 1989;107: 816-9.

11 Arden GB, Barrada A, Kelsey J. A new clinical test of retinal function based upon the standing potential of the eye. $B r F$ Ophthalmol 1962;46:449-67.

12 Yonemura D, Kawasaki K. New approaches to ophthalmic electrodiagnosis by retinal oscillatory potential, druginduced reponses from retinal pigment epithelium and cone potential. Doc Ophthalmol 1979;48:163-222.

13 Berson EL. Retinitis pigmentosa: the Friedenwald lecture. Invest Ophthalmol Vis Sci. 1993;34:1659-1676.

14 Yonemura D, Kawasaki K, Yamamoto SM. Hyperosmolarity response of ocular standing potential as a clinical test for ity response of ocular standing potential as a clinical test for
retinal pigment epithelium activity: chorioretinal dystroretinal pigment epithelium activity: ch
phies. Doc Ophthalmol 1984;57:163-173. 